\title{
Coulisses
}

Revue de théâtre

14 | Printemps 1996

Varia

\section{Le Théâtre de Tchekhov}

\section{Serge Rolet}

\section{OpenEdition}

Journals

Édition électronique

URL : http://journals.openedition.org/coulisses/4505

DOI : $10.4000 /$ coulisses.4505

ISSN : 2546-9460

\section{Éditeur}

Presses universitaires de Franche-Comté

\section{Édition imprimée}

Date de publication : 1 mai 1996

Pagination : 14-21

ISSN : 1150-594X

\section{Référence électronique}

Serge Rolet, «Le Théâtre de Tchekhov », Coulisses [En ligne], 14 I Printemps 1996, mis en ligne le 20 mars 2019, consulté le 17 juin 2020. URL : http://journals.openedition.org/coulisses/4505; DOI : https://doi.org/10.4000/coulisses.4505

Ce document a été généré automatiquement le 17 juin 2020.

Coulisses 


\section{Le Théâtre de Tchekhov}

\section{Serge Rolet}

1 Dans l'itinéraire artistique de Tchekhov, les grandes pièces sont tardives : la première, La Mouette, est de 1896. J'appelle "grandes pièces" les quatre dernières œuvres théâtrales de notre auteur, c'est-à-dire La Mouette, Oncle Vania, Les Trois Sceurs, et La Cerisaie. Selon certains spécialistes russes, il est légitime de considérer que ces quatre pièces constituent à elles seules «le » théâtre de Tchekhov : les autres titres, Platonov ou Ivanov, les petites pièces en un acte ne représentent que des esquisses, des galops d'essai. Le théâtre est donc pour Tchekhov l'achèvement de son activité artistique : son sommet et sa fin.

2 Tchekhov, pendant longtemps, n'envisage pas d'écrire pour le théâtre, en tout cas d'écrire autre chose que des petites pièces comiques, comme L'Ours ou La Demande en Mariage. Ses premiers textes, « humoresques ", parodies, etc., appartiennent à peu près tous au genre du récit.

Pourtant Tchekhov avait depuis l'enfance l'amour du théâtre. Des troupes, russes ou étrangères, venaient en tournée à Taganrog, sa ville natale ; le jeune Tchekhov assistait aux spectacles. À la maison, il jouait avec ses amis dans un petit théâtre, et c'est lui qui écrivait les pièces (des vaudevilles et des drames).

Comment comprendre que Tchekhov se soit longtemps attaché principalement au genre épique, au récit? En quoi ses pièces tranchent-elles sur le théâtre russe de son temps?

\section{La scène russe à l'époque de La Mouette}

\section{La « décadence de l'art théâtral »}

Quand Tchekhov commence à publier, au début des années 1880, le genre dramatique en Russie est peu dynamique, peu créatif; depuis les grandes pièces d'A. Ostrovski, il ne se passe pas grand-chose. Après quelques tentatives de donner à Pétersbourg et à Moscou quelques vaudevilles et la pièce Ivanov, Tchekhov comprend combien le théâtre russe traditionnel, surtout dans la mise en scène et le jeu des acteurs, est arriéré, 
stéréotypé. "Le théâtre d'aujourd'hui, c'est une éruption de boutons, une mauvaise maladie des villes. Il faut s'en débarrasser à coup de balai, mais il n'est pas sain de l'aimer ».

Après la première de La Mouette, et quelquefois sans plaisanter, Tchekhov écrit que le théâtre ne lui réussit pas, qu'il n'est pas fait pour devenir un auteur dramatique : «Le théâtre me porte si peu chance, si peu chance, que si je me mariais avec une actrice, l'enfant qui nous naîtrait aurait tout de l'orang-outang ou du porc-épic » (1898).

Tchekhov n'est pas le seul à penser ainsi. En 1897 se réunit le Congrès Théâtral panrusse. L'un des intervenants, le metteur en scène du Théâtre Maly A. Lenski, dans une communication intitulée Les causes de la décadence de l'art théâtral, affirme la nécessité de réformes radicales pour relever le niveau de la scène russe. C'est à cette époque qu'apparaitt la Société d'Art et de Littérature de Stanislavski. En 1898 est fondé le Théâtre d'Art de Moscou, qui réunit les troupes de Stanislavski et de NemorovitchDantchenko; le jeune Meyerhold en fait partie.

5 Les grandes pièces de Tchekhov coïncident donc avec un grand mouvement de rénovation du théâtre russe, dont l'initiative revient plus aux metteurs en scène qu'aux auteurs. La « décadence » en effet n'est pas tant celle du répertoire que celle de la scène proprement dite.

\section{Le répertoire}

6 Que joue-t-on alors en Russie ? On joue des pièces classiques, antiques (Sophocle), on joue Shakespeare. Le théâtre moderne n'est pas absent: la trilogie historique d'A.K. Tolstoï (Alexeï Konstantinovitch, qu'il ne faut pas confondre avec Lev Nikolaïevitch, auteur de Guerre et Paix, ni avec Alexeï Nikolaïevitch, romancier des années 1910-1920 !), grand succès du siècle, est toujours montée; on donne aussi des pièces étrangères (Ibsen, Hauptmann, Schnitzler, Maeterlinck).

Parmi les textes russes contemporains de La Mouette, on trouve la pièce de Lev Tolstoï L'Empire des Ténèbres (1887). Gorki, célèbre depuis 1892 pour ses récits, dont deux volumes paraissent en 1898, donne sa première grande pièce, Les Petits Bourgeois, en 1901, presque en même temps que Les Bas-Fonds, adaptés assez librement au cinéma par J. Renoir, avec Louis Jouvet dans un des rôles principaux.

La doctrine dominante, c'est l'utilitarisme esthétique. L. Tolstoï publie l'article Qu'est-ce que l'art? en 1897 : l'art est un moyen d'éducation morale. L'échec, en 1900, de la mise en scène, pourtant parfaitement maîtrisée et très belle, de La Fille des Neiges d'Ostrovski inspire à Meyerhold ce commentaire plein de regrets (dans une lettre à Tchekhov) : " Visiblement, dans la période troublée qui est la nôtre, alors que s'écroulent les structures de toute notre vie, il ne suffit pas d'en appeler à la seule beauté".

Il faut tempérer ce jugement de Meyerhold. À partir de 1898 souffle sur la Russie le grand vent du symbolisme. Volynski publie un article théorique important: Le symbolisme et les symbolistes, la revue La Balance commence à paraître. Ce mouvement, qui renouvelle complètement l'univers littéraire, s'inscrit entre autres en réaction contre l'utilitarisme esthétique. Tchekhov pouvait sur ce point voir en lui un allié ; sur le plan artistique, il avait avec les symbolistes beaucoup d'affinités. La beauté, avec ce nouveau courant poétique, retrouve ses droits, et il est possible, il devient même actuel $\mathrm{d}$ '« en appeler à elle ». 


\section{La scène}

7 Quoi qu'il en soit, la faiblesse du théâtre en Russie dans les années 1880-1890, c'est la scène : la formation de l'acteur, la tradition des «emplois » figés, la routine dans le jeu, la mise en scène, l'interprétation. Pour sortir le théâtre russe de l'ornière, Tchekhov écrit des pièces qu'il sera impossible de jouer comme on avait l'habitude de le faire. Les textes de Tchekhov exigent un saut décisif, et ce saut, il n'est guère que le Théâtre d'Art qui soit capable de l'exécuter.

L'accueil réservé à la première mise en scène de La Mouette donne une idée de ce à quoi le public était habitué. L'affiche, réalisée dans le style du spectacle de divertissement, annonçait une comédie (ce dernier point correspondait à la volonté de l'auteur), et le public était d'autant plus disposé à rire que la soirée était donnée en l'honneur d'une actrice de boulevard (E. Levkeïeva). Les gens s'attendaient à un spectacle léger, et s'efforçaient de rire à la moindre sollicitation: un des personnages sortait-il une tabatière de sa poche pour proposer du tabac à quelqu'un, la salle éclatait de rire. Mais comme les vraies occasions de s'esclaffer étaient plutôt rares, les spectateurs sont restés sur leur faim, et sont repartis mécontents. Les acteurs connus, qu'ils étaient venus voir faire leur numéro habituel, n'avaient guère trouvé à exprimer leur «talent». Il y a bien un comique Tchekhovien, simplement ce n'est pas du tout le même que celui de boulevard (Tchekhov appelle «comédie » ou même "vaudeville " telle ou telle de ses pièces, mais aucune ne contient de quiproquos, de retournements de situations, de chasses-croisés, d'imbroglios de situations, ni d'une manière générale de tous ces effets dont regorge la comédie boulevardière). Bref, l'intérêt de la pièce de Tchekhov n'est pas aperçu : le niveau artistique du Théâtre Alexandrinski est trop bas pour que La Mouette connaisse le succès qu'elle mérite.

\section{Un théâtre d'états d'âmes}

8 Le nouveau théâtre apparaît à Tchekhov comme théâtre de l'actualité, exempt de tout détour historique, débarrassé du pittoresque traditionnel :

Votre théâtre ne doit monter que des pièces contemporaines, uniquement cela! Vous devez traiter de la vie contemporaine... telle qu'elle est vécue par l'intelligentsia, celle dont il n'est pas question dans les autres théâtres, en raison de leur absence totale d'intellectualité et, en partie, de leur absence de talent.

Ce qui intéresse Tchekhov, tout au long de sa carrière d'écrivain, ce ne sont pas tant les anecdotes qu'il raconte (en dehors de leur charge humoristique), l'intrigue ou le déroulement de l'action au théâtre. Dans ses grandes pièces, il n'y a pas d'action. Le temps semble s'écouler sans ruptures, sans accélérations : il ne se passe rien. Le sujet, l'action, comptent moins que les conversations des personnages. Tchekhov rejette les péripéties comme non vraies. Il faut, dit-il, s'en tenir à Piotr Semionovitch qui épouse Maria Ivanovna. D'où le refus des prouesses historiques, des grands personnages, le refus des héros.

Dans Les Trois Sœurs, l'action ne progresse pas : les trois filles de général ne partent pas pour Moscou, et on comprend bien vite qu'elles n'y partiront jamais. Le seul événement sensationnel, le meurtre du baron Tusenbach, ne change rien à la situation.

Dans La Cerisaie, l'action s'arrête dès l'acte III (une fois que la vente de la cerisaie est un fait acquis). La poursuite de la pièce repose sur d'autres éléments. 
11 Si, d'une manière générale, il n'y a pas chez Tchekhov d'intrigue, c'est que les personnages n'ont pas d'adversaires ou d'ennemis; le « conflit », le déséquilibre, gagne l'ensemble des acteurs ; l'origine du mal n'est pas un individu, une volonté particulière, mais la tournure des choses, la manière dont cela se passe. Rien de tangible, de réel, n'est en cause. Voilà pourquoi les «emplois » sont inutiles, pourquoi la formation traditionnelle de l'acteur n'a plus de pertinence.

\section{L'impartialité de l'auteur et la continuité prose-théâtre}

Dans les nouvelles, le texte d'auteur est limité au maximum. Ce sont les personnages qui parlent, ce sont eux qui exposent la situation. L'auteur reste à l'écart. «Il vaut mieux éviter de décrire l'état d'âme des héros. Il faut essayer de les rendre perceptibles par les actes des héros ». Une fois libérés de l'intervention de l'auteur, les personnages deviennent plus communicatifs, plus sincères, et le lecteur a la possibilité d'approcher d'eux de plus près et de les comprendre mieux.

Le système de Tchekhov est fondé sur le lyrisme (le rire, la tristesse : autant d'émotions partagées). Le principe épique, même dans ses récits, lui est au fond étranger. Il n'y a rien d'étonnant à ce que, du récit court, Tchekhov soit passé au théâtre plutôt qu'au roman : du fameux roman, auquel il travaille de longues années, il ne reste finalement qu'une seule phrase : "Ils s'aimèrent, se marièrent, et ne furent pas heureux ».

Le lyrisme se manifeste surtout à partir du moment où les personnages montent sur la scène; dans les nouvelles, la structure du genre, probablement, limite l'épanchement lyrique (la nouvelle montre les personnages avant tout de l'extérieur). À propos du théâtre de Tchekhov, on a beaucoup parlé de "sous-texte lyrique ", pour essayer de rendre compte du fait que, dans les conversations les plus banales, il y avait du sens, que dans le trivial, il y avait de la poésie. Nous savons que l'abolition de l'opposition entre la "vraie vie » (la vie intérieure, celle qui possède un sens) et la vie ordinaire est un des projets de Tchekhov, l'un des points où il s'est séparé de la "grande» littérature.

\section{La révélation}

13 Tchekhov n'a pas le projet de montrer les actes de tel ou tel personnage, mais de décrire la conscience que ce personnage a de ses actes, la représentation qu'il bâtit de sa propre expérience. Décrire comment les personnages se débattent dans un monde auquel ils ne comprennent rien : voilà ce que veut faire Tchekhov.

Cette saisie particulière de la conscience des personnages se met en place dans les premières œuvres (non théâtrales) et s'affine au cours des années. Parmi les premiers textes de Tchekhov, datant d'avant 1888, l'année charnière qui le voit passer - comme malgré lui - de l'activité de journaliste humoristique à celle d'écrivain en vue, on distingue un groupe de nouvelles particulièrement importantes où se forge la vision du monde qui imprègne les œuvres plus tardives.

Ces nouvelles ont un schéma narratif caractéristique. Les héros en sont des « hommes du commun ", dont les intérêts se bornent à la vie de tous les jours. Soudainement ces personnages médiocres sont tirés de leur existence routinière par un événement inattendu. Cet événement non plus n'a pas de grandeur : il s'agit le plus souvent d'un détail trivial, d'un événement insignifiant ; mais il a l'effet d'un détonateur, il provoque une «révélation ». Le personnage voit s'écrouler toute sa représentation du monde, 
qu'il croyait pourtant stable et définitive. Le monde tel qu'il se l'imaginait apparaît dans sa fausseté, et la vie se montre dans une lumière nouvelle, son ordre « naturel » se fait jour. "Ordre », c'est un mauvais terme : le monde est confus, complexe, conflictuel. Le héros se met à réfléchir, mais il a perdu ses repères.

La révélation, la découverte, chez Tchekhov, a quelque chose d'original par rapport à ce qu'elle est chez Tourgueniev ou Tolstoï. Chez ces derniers, il y a aussi des découvertes, des révélations, mais elles sont présentées comme l'aboutissement d'un cheminement intérieur, comme un achèvement. Chez Tchekhov, au contraire, l'illumination n'est souvent qu'un éclair, le savoir, la nouvelle conscience des choses, peuvent disparaître, laissant le héros à ses interrogations, à son inquiétude, à son angoisse, mais aussi bien à la routine, à l'absurdité. L'oncle Vania, Voönitski, se révolte contre son beau-frère, le professeur d'université; il va jusqu'à lui tirer dessus à coups de revolver (scène de comédie s'il en est), mais une fois passé ce moment d'égarement, il se remet à travailler durement pour son compte : il n'y a, croit-il, rien d'autre à faire. La prise de conscience est comme un éclair, il n'en reste rien. La pièce est un cercle.

Décrire les états de conscience, et aborder à cette occasion des questions philosophiques, ou, au moins, donner au texte (ou à la pièce), une tonalité philosophique, voilà qui est courant chez les écrivains du XIX siècle; mais à la différence de ses contemporains et devanciers, Tchekhov ne suit jamais dans ses pièces (ni d'ailleurs dans ses récits) une doctrine philosophique ; s'il fait allusion aux théories en vogue, c'est, toujours, pour les tourner en ridicule. Il n'y a pas, chez lui, de pièce « à thèse ", comme chez Tolstoï, Gorki, ou, plus près de nous, chez Sartre.

Même quand un personnage est présenté comme sympathique, et que ses idées peuvent paraître a priori justes et fondées au lecteur, Tchekhov prend toujours ses distances avec le personnage et avec sa vision du monde. Les héros de Tchekhov sont constamment caractérisés comme incapables de s'orienter dans le monde, de comprendre correctement ce qui leur arrive.

Cette absence de solidarité entre l'auteur et les personnages se manifeste de plusieurs façons. Tout d'abord, les personnages font leur propre critique; ils s'accablent euxmêmes des reproches les plus fondés, reconnaissent leurs torts, jettent un doute, à un moment ou à un autre, sur leurs convictions. Ils avouent qu'ils ne savent pas. Dans Oncle Vania, le médecin, Astrov, personnage sympathique et grand séducteur, ne peut expliquer pourquoi il s'est laissé gagner par la vulgarité, il n'a pas d'idée pour changer ce que sa vie a de médiocre, de laid. Chez lui, l'incapacité à s'orienter dans l'existence revient sans cesse, elle constitue un trait majeur du personnage. Tchekhov fait aussi coexister dans le texte, celui de la pièce comme celui de la nouvelle, des vérités qui s'excluent mutuellement, sans indiquer si l'une a sa faveur plus que l'autre: toutes les deux se détruisent. Il arrive que les personnages au départ les plus sympathiques, se montrent sous un jour finalement peu flatteur, ou l'inverse. Lopakhine dans La Cerisaie, se montre pathétique et odieux; le jeune écrivain à succès dans La Mouette, lui, est ambigu, on ne sait trop comment l'apprécier. Ni l'un, ni l'autre ne bénéficie de la solidarité de l'auteur, Tchekhov, en tout cas, ne parle pas par leur bouche.

Et puis, il y a l'épreuve des faits, la destruction du milieu au sein duquel, longtemps, l'illusion a pu être préservée. Dans La Cerisaie, les propriétaires du verger, dont on mentionne l'existence jusque "dans l'Encyclopédie», ne comprennent pas que le temps de la noblesse terrienne est passé, que la perpétuation des traditions ne pourra rien contre les dettes accumulées au cours d'années de voyages et d'oisiveté.

L'incompréhension se traduit par l'inaction, et par le fait que les personnages ne sont 
tournés que vers le passé, vers l'enfance (symbolisée par cette armoire dont, à la fin de la pièce, tombe une poussette). Malgré toutes les paroles échangées, les confessions à cœur ouvert, les reproches et les bonnes résolutions, le verger ne sera pas sauvé : quand le rideau tombe, le spectateur entend les coups de hache qui s'abattent sur les arbres du domaine. Les personnages ne sont pas crédibles.

\section{L'émotion}

Il faut donc prendre garde à la distance que Tchekhov met entre lui (et du même coup, entre nous) et ses héros. Mais il n'empêche : les personnages, si faibles, si désarmés face à l'absurdité de leur existence sont à la fois ridicules et pathétiques. Les discours des personnages ont la part belle. Les rêves et les illusions des personnages occupent le devant de la scène ; la sanction n'arrive qu'in extremis. L'émotion, le lyrisme sont tels que le spectateur ne perçoit plus vraiment de critique. Tchekhov n'insiste pas sur la fausseté des représentations, sur leur ridicule ; il les suggère simplement. La distance, l'ironie, si visibles dans les nouvelles, sont à ce point atténuées dans le théâtre de Tchekhov que le spectateur s'identifie aux personnages, se laisse gagner par la tristesse, la nostalgie. Les personnages ont beau être dérisoires, ils sont tout de même sublimes (Macha, par exemple, dans Les Trois Souurs). Ce n'est pas commettre de contresens que de pleurer en regardant une pièce de Tchekhov. À cet égard on peut comparer Tchekhov à Flaubert : le « romantisme » de Madame Bovary est ridicule, mais la vie n'a rien d'autre à lui offrir, à nous offrir.

Les trois sœurs, 1901

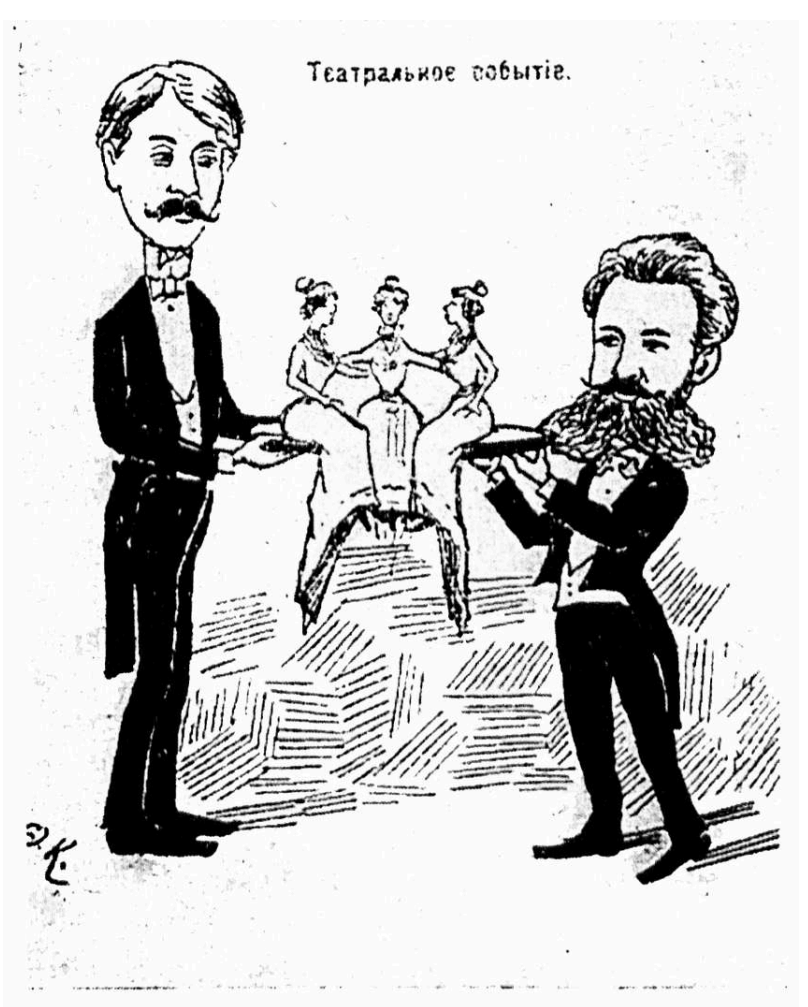

D'ailleurs souvent, par exemple dans La Cerisaie, la sanction, l'épreuve des faits, se révèlent finalement moins terribles qu'on pourrait le supposer : au milieu de la gravité, 
de la douleur, se fait jour quelque chose de léger, et la pièce, sensiblement, retrouve le ton de la comédie. Dans La Cerisaie, plusieurs personnages descendent des figures comiques des petites nouvelles : Epikhodov, Simeonov-Pichtchik, Charlotte. SimeonovPichtchik, par exemple, explique qu'il a pour ancêtre le cheval que l'empereur Caligula avait nommé consul. Lui-même, Tchekhov était persuadé d'avoir écrit des pièces franchement comiques. Rappelons sa mauvaise humeur après les premières représentations des Trois Sœurs : la pièce était d'après lui une pure comédie, et il était furieux que le public n'ait retenu que la gravité, le tragique de la situation.

Encore un mot sur la «légèreté ». Quelquefois, il n'y a pas de sanction vraiment brutale: l'inefficacité des personnages à conduire correctement leur vie est hors de doute, mais elle n'est pas " prouvée » par un événement, par les faits (tel est le cas dans Les Trois Sœurs). L'illusion semble pourtant pouvoir durer toujours.

Le message contenu dans les pièces de Tchekhov est donc complexe, ambigu. La nostalgie de l'enfance perdue est ridicule, mais elle est belle, et il n'y a peut-être rien d'autre qui puisse donner la paix. Voilà ce que le spectateur peut se dire. À moins qu'il ne réagisse comme Gorki, gêné par l'absence de clarté de La Cerisaie :

J'ai écouté la pièce de Tchekhov. A la lecture, elle ne fait pas l'impression d'une pièce importante. Pas un seul mot de nouveau. Tout, les étals d'âme, les idées (si on peut encore parler d'idées), les personnages, tout ça était déjà dans ses autres pièces. Évidemment, c'est beau, et bien entendu, le public va être gagné par le spleen le plus noir. Mais pourquoi le spleen? Ça je ne sais pas.

Pourquoi avoir risqué de dérouter les spectateurs, de les laisser se perdre dans des interprétations trop complaisantes à l'égard des héros, par exemple ? Pourquoi, si ce que disent les personnages est si vain, s'y attarder au point qu'on se laisse entraîner par le charme de ces paroles, de ces voix nostalgiques et douces?

Évoquons rapidement la voie que Tchekhov n'a pas choisie, celle de Gorki. Tchekhov aurait pu incarner ses positions dans un personnage, comme Gorki en Nil, dans Les Petits Bourgeois, ou en Satine, dans Les Bas-Fonds. Le message, peut-on croire, aurait été plus « clair ». En fait, rien n'est moins sûr. La position de Gorki dans Les Petits Bourgeois a été bien comprise par le public, mais ce n'est pas le cas avec Les Bas-Fonds. Le malheur de cette pièce, montée par Stanislavski au Théâtre d'Art de Moscou, c'est que le public a été séduit par le message religieux de Lucas, et non par celui, révolutionnaire, de Satine! Cela prouve qu'une "pièce à thèse " peut parfaitement donner lieu à un contresens.

Cela dit, si Tchekhov a préféré la complexité, l'absence de clarté du message que portaient ses pièces, c'est pour des raisons de fond. Il faut maintenant aborder l'une des grandes convictions artistiques - et aussi philosophiques - de Tchekhov, celle qui lui fait refuser les vérités générales. Dans l'article Tchekhov et son temps ${ }^{1}$ nous avons vu qu'il considérait que l'artiste n'avait pas à prescrire de solution aux problèmes posés par ses œuvres. Peut-être faut-il aller un peu plus loin, et voir quelle idée Tchekhov se faisait de la vérité. 


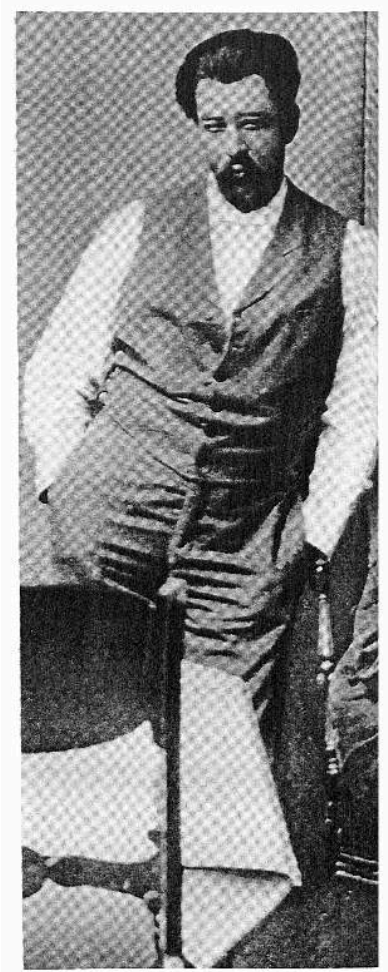

\section{« Expliquer chaque événement en particulier »}

17 Le travail de Tchekhov, ainsi qu'il l'explique lui-même dans sa correspondance, s'organise autour de plusieurs mots d'ordre : le refus des "vérités spéciales », la nécessité de "poser correctement les problèmes", le désir d'«expliquer chaque événement en particulier ", l'opposition entre "idée générale » et "vérité réelle ».

Tchekhov porte une grande attention aux circonstances dans lesquelles une pensée s'élabore. Les circonstances ne sont jamais "secondaires": la pensée "juste», «noble » et « sincère » exprimée par un personnage est en grande partie le résultat des circonstances; l'élan le plus enthousiaste n'est - peut-être - que la conséquence d'une émotion passagère. Dans Une Histoire Sans Fin, ce qui pousse le héros au suicide c'est, bien sûr, sa pauvreté et la mort de sa femme, mais c'est tout autant les effets de lumière de la chandelle. La révolte de Voïnitski contre le professeur tient peut-être plus à la jalousie (il est amoureux de la jeune femme de ce dernier), à quelque chose d'irrationnel, de contingent, qu'à la maturation enfin achevée de sa " conscience ».

Dans la poétique de Tchekhov les détails ont un statut très particulier. Dans le monde de Tchekhov, ce qui constitue la réalité n'est pas hiérarchisé ; l'accessoire, le trivial, sont traités de la même façon que 1 '« essentiel». L'accessoire vaut autant que le principal. Entre les deux, la différence s'efface. Pourquoi cette obstination à refuser de hiérarchiser ? La raison en est le primat donné à l'individu, la méfiance à l'égard des idées générales, de l'abstraction, de l'universel. Il faut se souvenir que Tchekhov était médecin. À l'école des grands médecins russes à la fin du XIX ${ }^{e}$ siècle, principalement du professeur Zakharine, Tchekhov s'est convaincu que seule l'attention passionnée accordée à l'ensemble des éléments d'une situation permet de comprendre «ce qui se 
passe ». Le modèle de la science, c'est celui de la biologie, de la médecine, non plus de la physique. Les relations entre les hommes ne sont pas régies par une loi, ni même par plusieurs, censées déterminer "verticalement" les choses. Ces relations, mieux vaudrait les concevoir à la manière d'un être organique, construit « horizontalement »: un petit stimulus, une petite modification, peut modifier l'ensemble du système. La bonne méthode en médecine se révèle également bonne dans la vie : Zakharine insistait beaucoup sur ce point. Tchekhov se montre bon disciple. Pour lui ce qui pourrait paraître contingent, sans importance, est justement ce qui rend chaque objet unique, profondément individuel. Le détail sans signification apparente ne peut être écarté d'une caractérisation qui se veut correcte, qui vise la vérité réelle, non la vérité abstraite, théorique.

On comprend dès lors plus facilement cet autre refus de Tchekhov, celui de la vérité spécialisée, du savoir spécialisé. Ce n'est pas du tout un refus de la science, de la raison, ou toute autre attitude obscurantiste de ce genre. Le savoir spécialisé est fragmentaire ; il décrit un objet sous un certain angle, indépendamment du tout complexe auquel il appartient, il en élimine les détails, de manière artificielle. Le "tuyau de descente », c'est la polarisation appauvrissante de l'esprit sur un aspect isolé de la réalité ; dans ce cas, pour Tchekhov, même l'exactitude, même une certaine « vérité » sont inutiles.

Considérons maintenant les personnages de Tchekhov, les idées qu'ils expriment. Chacun d'eux voit le monde de manière trop étroite, sous un seul angle: tous, ils s'en tiennent aux « savoirs spécialisés ", c'est pourquoi ils ne "posent pas correctement les problèmes ». Prisonniers des vérités particulières, ils ne se parlent pas vraiment. Chacun s'exprime indépendamment $d u$ discours des autres. Cet aspect, l'incommunicabilité des consciences enfermées dans leur vision particulière, est l'un des traits les plus modernes des pièces de Tchekhov : nous sommes tout près du théâtre de l'absurde. À cette différence - majeure - près que Tchekhov n'affirme pas lui-même que la vie n'a pas de sens: ce sont ses personnages qui nous donnent cette idée. Tchekhov nous invite à considérer la situation dans sa globalité, à entendre les voix des personnages toutes ensemble, à ne pas tomber dans le même travers que ses héros. Tout comme les détails tirés du monde des objets, du décor, ne peuvent pas être isolés les uns des autres, ni de l'ensemble auquel ils appartiennent, il faut se garder de considérer les personnages Tchekhoviens séparément. Parmi les circonstances et les détails qui les enveloppent, il faut compter les paroles et les pensées, les gestes des autres personnages. Alors seulement il est possible qu'un sens se fasse jour : à chacun de nous de dire lequel. Tchekhov, quant à lui, n'affirme rien.

Si tout est lié, les éléments contenus dans une pièce peuvent entrer dans des rapports nombreux, subtils; chaque metteur en scène peut donner libre cours à sa liberté d'interprétation. Si les éléments ne sont pas hiérarchisés, si, en même temps, on peut les supposer a priori hétérogènes, ils sont peut-être liés par quelque rapport caché, le texte dramatique se met à résonner d'une infinité de sens possibles. La poussette qui tombe de l'armoire dans La Cerisaie, ce n'est pas simplement une poussette qui tombe d'une armoire ; l'objet est relié d'une manière particulière aux autres objets, aux mots, aux accents de la pièce. Tout cela est très subtil. R. Grenier, dans sa préface à un recueil de nouvelles de Tchekhov, avoue ne pas bien comprendre la signification du fait que, chez Tchekhov, un « sec coup de fusil » peut « changer un peu l'âme » des personnages, « la rendre meilleure ». Ce qui, probablement, est en jeu dans cet exemple, c'est la vision non hiérarchique de la réalité, l'idée que des rapports existent entre des éléments du réel, et que nous ne les connaissons peut-être pas. 


\section{Tchekhov et le Théâtre d'Art de Moscou}

\section{Le Théâtre d'Art : une chance pour Tchekhov}

"Je remercie le ciel d'être tombé sur cette île merveilleuse qu'est le Théâtre d'Art, alors que je voguais sur la mer du quotidien ». Tchekhov, on l'aura compris, doit beaucoup au Théatre d'Art, à ses dirigeants et à ses acteurs. Ce n'est qu'avec le Théâtre d'Art que le théâtre d'états d'âmes devient accessible au public, que Tchekhov, enfin, est reconnu pour ce qu'il est. Le talent de Stanislavski comme acteur, la hauteur de ses exigences à l'égard des autres acteurs permettaient de sortir de la routine symbolisée par «l'entreprise des spectacles" qu'était devenu le prestigieux Théâtre Maly de Moscou. Tchekhov n'a collaboré à aucun autre théâtre de manière aussi régulière et approfondie qu'avec le Théâtre d'Art. Longtemps, la doctrine de Stanislavski a fait autorité dans les mises en scène de Tchekhov (comme de bien d'autres auteurs...). Sans vouloir nier la valeur de l'enseignement de Stanislavski en général, ni son rôle dans l'épanouissement du théâtre de Tchekhov dans les années 1900, et jusqu'à aujourd'hui, je voudrais simplement apporter un correctif à cette idée tout de même un peu trop simple, selon laquelle «Tchekhov, c'est Stanislavski ».

\section{Les deux voies concurrentes suivies par le Théâtre d'Art}

Il y avait, dans le Théâtre d'Art de Moscou, deux tendances opposées, et, si l'on en croit Vsevolod Meyerhold, orfèvre en la matière, l'une de ces tendances, celle qui poussait le travail théâtral du côté du naturalisme, était opposée à ce que les pièces de Tchekhov ont de plus précieux : le théâtre d'états d'âmes, le lyrisme, le rythme, la musique.

La tendance naturaliste, inspirée par les Meininger, est représentée au sein du Théâtre d'Art par Stanislavski ; Nemirovitch- Dantchenko, lui, selon l'expression de Meyerhold, "défend les intérêts de l'auteur", dans le cas qui nous occupe, ceux de Tchekhov. Dans le travail du Théâtre d'Art sur les textes de Tchekhov, nous trouvons les éléments d'un désaccord entre Stanislavski et Tchekhov. Leur divergence de vues est celle qui sépare le naturalisme de ce qu'on a appelé le « théâtre de la convention ».

On connaît assez l'influence de Stanislavski sur le destin du théâtre de Tchekhov; il semble qu'une influence tout aussi décisive, quoique moins célèbre, ait été exercée par Tchekhov sur le Théâtre d'Art.

Écoutons Meyerhold :

Cette circonstance qui permit au Théâtre d'Art d'abriter sous le même toit le théâtre naturaliste et le théâtre d'états d'âmes, je suis profondément convaincu que c'est Tchekhov lui-même qui a contribué à la créer, précisément parce qu'il assistait en personne aux répétitions de ses pièces et que, par le charme de sa personnalité comme par les fréquentes conversations qu'il avait en privé avec les acteurs, il a influencé leurs goûts et leurs attitudes à l'égard des problèmes esthétiques.

Meyerhold raconte comment les choses se passaient concrètement, pendant les répétitions :

Tchekhov assiste pour la seconde fois aux répétitions de La Mouette (11 septembre 1898) au Théâtre d'Art de Moscou. Un des acteurs lui raconte que dans cette pièce, derrière la scène, des grenouilles coasseront, des libellules striduleront et des chiens aboieront.

- Pourquoi tout cela? demande Anton Pavlovitch d'un air mécontent. 
- Cela fait réel, répond l'acteur.

- Cela fait réel, répète Anton Pavlovitch avec un sourire moqueur; et il ajoute après une petite pause: "La scène, c'est de l'art. Kramskoïa a magnifiquement représenté des visages, dans sa peinture de mœurs. Qu'adviendrait-il si, sur un de ces visages, on découpait le nez peint pour le remplacer par un vrai ? Le nez serait réel, mais le tableau serait gâché ».

Un des acteurs raconte (...) qu'à la fin du troisième acte de La Mouette, le metteur en scène veut amener sur le plateau toute la domesticité, notamment une femme avec un enfant en pleurs.

- Il ne faut pas. C'est comme si au moment où vous jouez pianissimo le couvercle du piano retombait.

- Dans la vie, il arrive souvent que dans le pianissimo fasse irruption un forte tout à fait inattendu pour nous, tente de plaider un des acteurs du groupe.

- Oui, mais la scène, dit Anton Pavlovitch, exige une certaine convention. Vous n'avez pas le quatrième mur. En outre la scène, c'est de l'art, la scène reflète la quintessence de la vie et il ne faut rien y introduire de superflu.

La Mouette. Mise en scène Théâtre d'art de Moscou. 1898

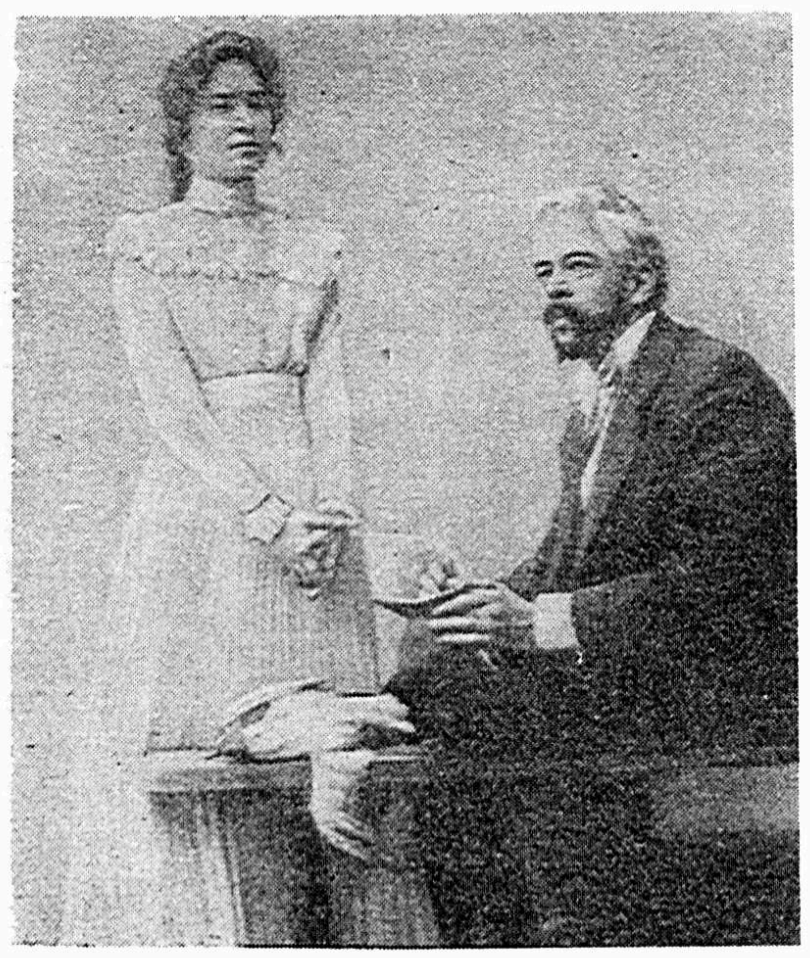

Trigorine, Stanislavski.

Meyerhold explique que le théâtre dut son nouveau visage à un groupe d'acteurs bien précis, qu'on appellera d'ailleurs « acteurs Tchekhoviens », avec lesquels l'auteur avait des relations particulièrement étroites, et qui ont préservé des accents non naturalistes dans les mises en scène de plus en plus soumises à l'orchestration vériste de Stanislavski.

En quoi le naturalisme est-il nocif aux pièces de Tchekhov? Parce qu'il les fragmente en une multitude de morceaux qui prennent chacun une valeur autonome. A force de développer telle ou telle scène secondaire, en appuyant les effets, en utilisant toute une machinerie, en accentuant le jeu des acteurs, les relations subtiles, incertaines, musicales, entre les éléments de la pièce finissent par se perdre. Le sous-texte est tué 
par l'irruption d'une réalité trop concrète.

La critique de fond de Meyerhold est la suivante :

Dans les pièces de Tchekhov, le particulier détourne le metteur en scène de $\mathrm{T}$ image d'ensemble, parce que les personnages, esquissés de manière impressionniste, constituent un matériau de choix dont on peut profiter pour pousser le tracé jusqu'à son achèvement en figures éclatantes (types).

Par exemple, le metteur en scène de La Cerisaie

a fait de l'épisode de prestidigitation une scène toute entière, avec tous les détails et tous les trucs, longue et compliquée. En concentrant là-dessus son attention, le spectateur perd de vue le leitmotiv de l'acte. Et si à la fin de l'acte les mélodies de fond restent dans la mémoire, le leitmotiv, enterré par la mise en scène, a disparu (...).

En fait, l'équilibre entre la convention nécessaire au théâtre d'états d'âmes et le traitement naturaliste des pièces de Tchekhov n'aura pas survécu à la période de mise en place des structures du Théâtre d'Art :

Le théâtre naturaliste se montra très persévérant dans sa volonté d'éliminer de la scène la puissance du Mystère. Ainsi, dans la première version scénique de La Mouette, au premier acte on ne voyait pas où s'en allaient les personnages quand ils quittaient le plateau. Après avoir traversé en courant un petit pont, ils disparaissaient dans la tache noire d'un fourré, quelque part (à cette époque, le décorateur du théâtre travaillait encore sans la collaboration du modeleur). Par contre, à la reprise de La Mouette, tous les coins du plateau étaient découverts (...). Dans la première version de La Mouette, au troisième acte, il y avait une fenêtre latérale, et on n'apercevait pas le paysage. Lorsque les personnages entraient dans l'antichambre avec leurs caoutchoucs, secouaient leurs chapeaux, leurs plaids et leurs châles, on imaginait l'automne avec sa petite pluie fine, les grandes flaques d'eau dehors et, pour les franchir, les caillebotis qui font floc. Mais à la reprise de la pièce, sur un plateau techniquement perfectionné, on avait percé des fenêtres en face des spectateurs. On voyait le paysage. L'imagination dès lors n'a plus qu'à se taire et, quoique disent les personnages à propos du paysage, on ne les croit pas, il ne peut pas ressembler à leur description: il est peint, on le voit. Et au final du troisième acte, dans la première version, le départ de la troïka tirée par des chevaux à grelots n'était que deviné derrière la scène et se dessinait d'autant plus vivement dans l'imagination du spectateur. Mais dans la seconde version le spectateur exige de voir ces chevaux à grelots, puisqu'on lui montre la véranda d'où partent les voyageurs. ${ }^{2}$

Voilà un aperçu du débat qui s'est développé autour de la mise en scène des pièces de Tchekhov, et qui (certains le regrettent) a vite tourné court: le charisme de Stanislavski a en effet conduit le jeune Meyerhold à poursuivre sa carrière en dehors du Théâtre d'Art.

Quoi qu'il en soit, j'espère que le lecteur trouvera là un élément qui lui permettra, si besoin est, de «contextualiser » quelque peu les préceptes de Stanislavski contenus dans « La formation de l'acteur ». 


\section{NOTES}

1. NDLR

2. (C'est toujours Meyerhold qui parle; tous les extraits de Meyerhold cités ici sont tirés d'Histoire et technique du théâtre, 1907, paru en français dans V. Meyerhold, Écrits sur le théâtre, Lausanne, 1973, traduction de B. Picon-Vallin). 